\title{
OCORRÊNCIA DO ORVALHO NA CIDADE DE SÃO PAULO
}

\author{
Gustavo ARMANI \\ Frederico Luiz FUNARI \\ Sergio Torre SALUM
}

\begin{abstract}
RESUMO
O presente trabalho analisa a ocorrência de dias com orvalho na cidade de São Paulo e a relaciona com as temperaturas do ar e do ponto de orvalho, bem como com as umidades relativa e absoluta do ar. Foram utilizados dados diários da Estação Meteorológica do Instituto de Astronomia, Geofísica e Ciências Atmosféricas (IAG) - USP, localizada no Parque Estadual Fontes do Ipiranga (PEFI), no período de 1958 a 2007. A partir de análises estatísticas de regressão linear e análise de variância (ANOVA), verificou-se, nestes 50 anos, aumento da frequência dos dias com orvalho, principalmente no outono, acompanhado de aumento da temperatura do ar e umidade absoluta $\mathrm{e}$ diminuição da umidade relativa.
\end{abstract}

Palavras-chave: orvalho, temperatura do ar, umidade relativa, clima urbano, São Paulo.

\begin{abstract}
This study analyzes the occurrence of days with dew in the city of São Paulo and correlates it with air temperatures and the dew point, as well as with the relative and absolute humidity of the air, based on daily data for the period from 1958 to 2007, obtained at the Meteorological Station of the University of São Paulo Institute of Astronomy, Geophysics, and Atmospheric Sciences (IAG-USP), Parque Estadual Fontes do Ipiranga (PEFI). Statistical analysis of linear regression and analysis of variance (ANOVA) show an increase in the frequency of days with dew, especially in autumn, as well as an increase in temperature and absolute humidity and a decrease in relative humidity during these 50 years.
\end{abstract}

Keywords: dew, air temperature, relative humidity, urban climate, São Paulo.

\section{INTRODUÇÃO}

O orvalho é um hidrometeoro proveniente da condensação do vapor d'água atmosférico sobre uma superfície sólida. Essa condensação inicia-se e se mantém quando a temperatura da superfície torna-se, ou permanece, igual ou inferior à temperatura do ponto de orvalho do ar adjacente. Esse fenômeno é distinto das gotas formadas por gutação.

De acordo com AYOADE (1988), existem dois processos básicos para a formação do orvalho: 1) sob calmarias, o vapor de água difunde-se do solo para a superfície exposta em processo de resfriamento radiativo. Quando a temperatura da superfície atinge ou se torna menor que a temperatura do ponto de orvalho, o vapor de água se condensa sobre a superfície; 2 ) sob condições de vento fraco, ocorre a transferência turbulenta de vapor de água da atmosfera para a superfície fria (igual ou menor que a temperatura do ponto de orvalho), formando o hidrometeoro sobre ela. Esse processo é também conhecido como orvalhada, sendo mais raro que o primeiro.

O orvalho é importante e fundamental nos países de clima quase desértico, como Israel, onde recipientes adequados são colocados, durante a noite, para a coleta da água proveniente da for- 
mação do orvalho. No deserto de Neguev, culturas agrícolas utilizam esse procedimento para irrigação. Entretanto, o orvalho é importante também nas regiões tropicais, porque a quantidade de água depositada é da ordem de $3 \mathrm{~mm}$ em uma só noite (ARGENTIERE, 1960). Infelizmente, trata-se de assunto ainda pouco investigado no Brasil.

Nessa perspectiva é que trabalhos como de DUVDEVANI (1947), MONTEITH (1957) e FRITSCHEN (1963) tomam importância quanto a aspectos teóricos e de mensuração do orvalho. No Brasil, FRANÇA (1946), estudando o clima da Bacia de São Paulo, relata, a partir de dados da Estação Meteorológica do IAG-USP, no período 1933 a 1942, que a maior frequência da ocorrência de orvalho se dava no final do outono. SERRA (1969), em seu Atlas Climatológico do Brasil, publicou mapas mensais de ocorrência de orvalho. Esses mapas são objeto de detalhada análise feita pelo mesmo autor, em 1977, quando destaca que as áreas onde o orvalho é mais frequente apresentam menor número de dias claros, sendo que ao contrário, os dias encobertos favorecem, devido ao menor aquecimento durante a tarde, a ocorrência de orvalho (SERRA, 1977).

MONTEIRO $(1976,2003)$ propõe o estudo do clima urbano por meio de três canais de percepção, sendo que o orvalho está inserido no canal III, referente ao impacto hidrometeórico. Como dificilmente o orvalho causa problemas na vida urbana em relação aos outros hidrometeoros do ambiente tropical, ele nunca foi estudado segundo a proposta feita por esse autor.

FUNARI et al. (2008) apresentam uma análise de 50 anos (1958-2007) da frequência mensal de dias com orvalho para a cidade de São Paulo com base nos dados da Estação Meteorológica do IAG-USP, constatando que os meses de maior número de dias com orvalho foram de abril a julho (outono).

O presente trabalho analisa a ocorrência de dias com orvalho (D) em São Paulo, relacionando-a com a temperatura do ar (T), a temperatura do ponto de orvalho (Td), a umidade relativa (UR) e a umidade absoluta (UA) para a cidade de São Paulo em uma série de 50 anos de dados diários (1958-2007).

\section{MATERIAL E MÉTODO}

Os dados diários de ocorrência de orvalho, temperatura do ar e do ponto de orvalho $\left({ }^{\circ} \mathrm{C}\right)$ e umidades relativa $(\%)$ e absoluta do ar $\left(\mathrm{g} . \mathrm{m}^{-3}\right)$ foram coletados na Estação Meteorológica do IAG-USP, instalada no Parque Estadual das Fontes do Ipiranga (PEFI), na zona sul da cidade de São Paulo (latitude: $23^{\circ} 39^{\prime} \mathrm{S}$, longitude: $46^{\circ} 37^{\prime} \mathrm{W}$, altitude: $799,22 \mathrm{~m}$ com registro na Organização Meteorológica Mundial - OMM, sob o número 83.004). Apenas para a temperatura do ponto de orvalho foi utilizada a série 1990-2007.

A observação de D é feita visualmente pelo observador meteorológico da estação. O observador anota sua ocorrência na folha de observação no espaço reservado para fenômenos diversos. O registro desse fenômeno é anotado e posteriormente revisado, sendo que as falhas de observação podem ser consideradas nulas, ou praticamente nulas. Como o período de observação da estação é das 07:00h às 24:00h, durante a madrugada, não há observação; caso, eventualmente, ocorra sua formação neste intervalo e ela se dissipar antes das 07:00h, a ocorrência do fenômeno não será registrada. Entretanto, dificilmente, o orvalho se formará de madrugada e se dissipará antes da primeira observação do dia.

Existem também na estação do IAG-USP dados sobre a quantidade de orvalho depositada ao longo do tempo registrado pelo orvalhógrafo, no período de 1958 a 1998, mas a consistência desses dados deve ser melhor analisada. Por isso, os dados do orvalhógrafo não foram utilizados neste trabalho.

De posse dos dados, foram elaboradas tabelas com o registro diário de orvalho (sim ou não) e dos totais mensais e anuais de dias de ocorrência de orvalho para a série 1958-2007.

Para se verificar a variação de D, T, Td, UR e UA com o tempo cronológico foi realizada uma análise de regressão linear pelo método dos mínimos quadrados, a partir dos dados diários. Para T, UR e UA, também, foram realizadas as análises de variância paramétrica (ANOVA) com a finalidade de se obter a significância estatística e a probabilidade de erro (p). Nos gráficos de tendência anual dos elementos, optou-se por realizar a apresentação sem os dados diários no intuito de não poluir visualmente os gráficos, muito embora as análises tenham sido conduzidas a partir dos dados diários. Foram elaborados vários histogramas de frequência de T, Td, UR e UA para se detectar quais classes são mais frequentes desses elementos nos dias com $\mathrm{D}$, e quais os meses com maior formação de D. Todos eles foram ajustados à curva de distribuição normal para se comparar com a distribuição real dos dados. 


\section{RESULTADOS}

A partir da distribuição da frequência de ocorrência de orvalho em função dos meses do ano, no período 1958-2007 (Figura 1), observase que a maior ocorrência do hidrometeoro é nos meses de abril, maio, junho e julho. É relevante observar que o orvalho é mais frequente nos meses de outono que nos de inverno. No inverno, normalmente, são esperados menores valores de Temperatura da Superfície (Ts), quando pode ser formado D. Entretanto, é preciso que exista suficiente umidade no ar para essa formação, do contrário Td será muito baixa (quanto maior UR maior Td). Nesse sentido, no inverno, quando a umidade do ar é normalmente menor, acredita-se que essa redução dificulte a formação do orvalho, pois $\mathrm{Td}$ é menor. Em outras palavras, como a $\mathrm{Td}$ é função de UR e de T, no inverno ela deve ser comumente menor que Ts, sendo que no outono Ts deve se igualar a Td com maior frequência. Ou seja, no outono, Ts é mais próxima de Td que no inverno. Estes resultados concordam com aqueles apresentados por SERRA (1977).

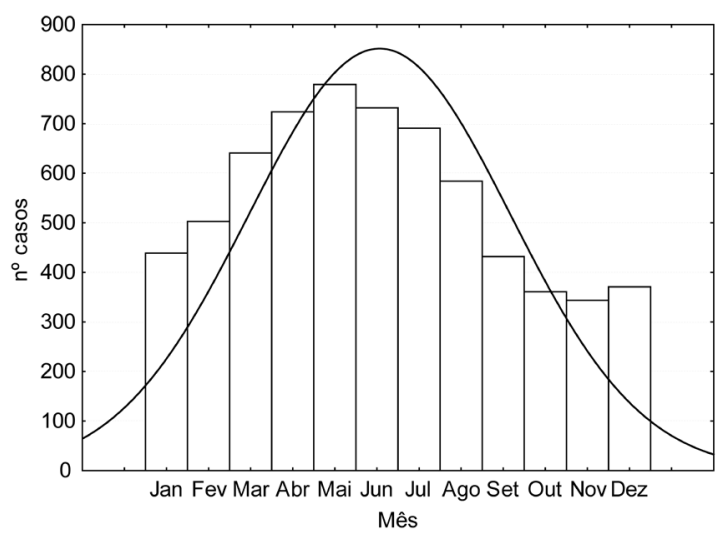

FIGURA 1 - Frequência de ocorrência de orvalho em São Paulo (série 1958-2007).

A frequência do número de dias de $\mathrm{D}$ no mesmo período (Figura 2), para as diversas faixas de UR (\%) média, máxima e mínima diária, mostra que a maior frequência de D ocorre quando a UR média está entre $80 \%$ a $86 \%$ (Figura 2a). Esse fato surpreende à primeira análise, pois pode- se pensar que a UR deva ser mais elevada para que se forme D. A umidade relativa muito alta indica condições de forte tensão de vapor, com perda de radiação noturna pouco intensa, dificultando a formação de D. Já com UR muito baixa, a queda noturna de temperatura, embora acentuada, não permite que T alcance Td. É justamente por isso que as mínimas diárias de umidade relativa, nos dias com formação de orvalho, não são tão baixas e as máximas também não são, comumente, saturadas (100\%). SERRA (1977) classifica a UR abaixo de $65 \%$ e acima de $85 \%$ como restritiva para a formação de orvalho, concordante com os resultados aqui apresentados para a cidade de São Paulo.
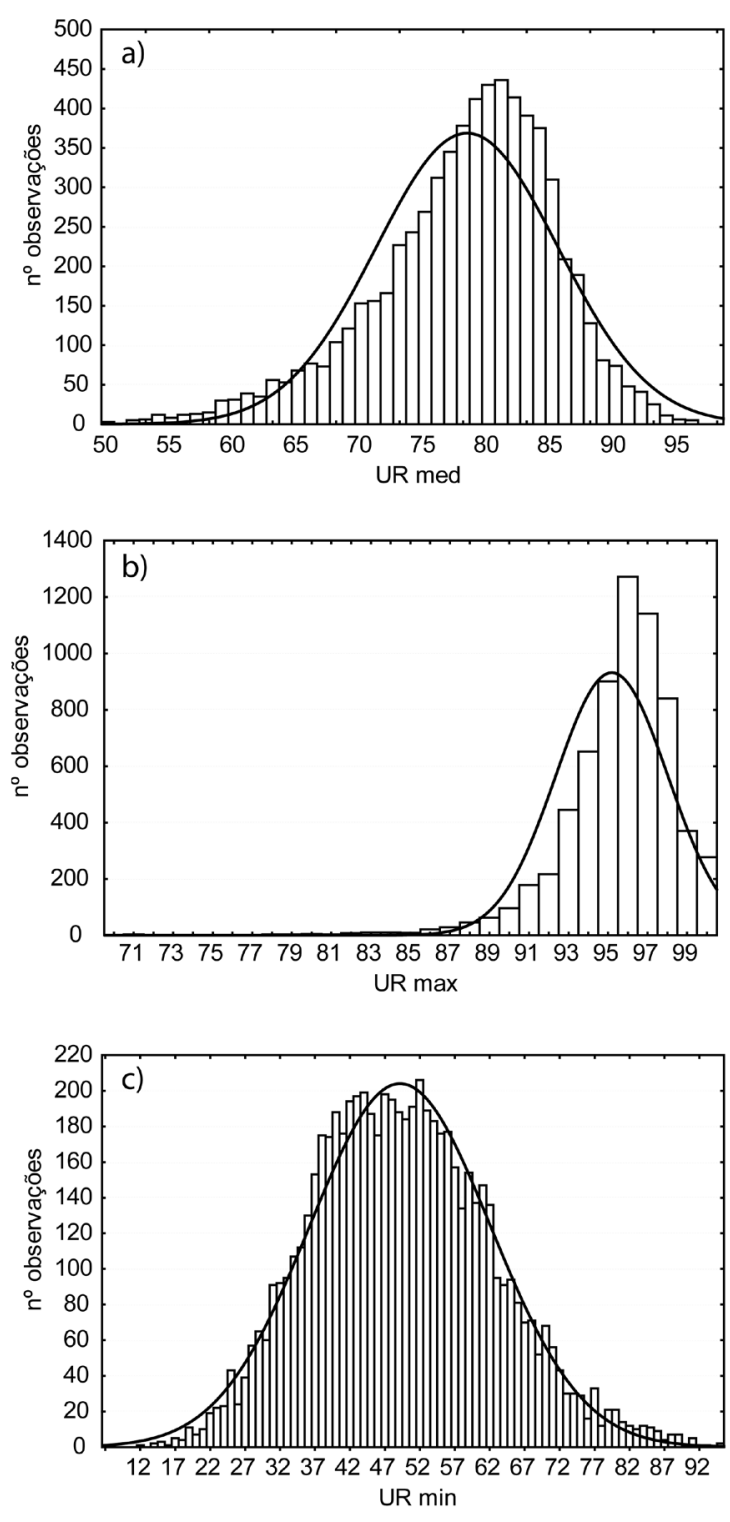

FIGURA 2 - Frequência de ocorrência de orvalho em São Paulo, segundo a umidade relativa: a) média diária; b) máxima diária; c) mínima diária (série 1958-2007). 

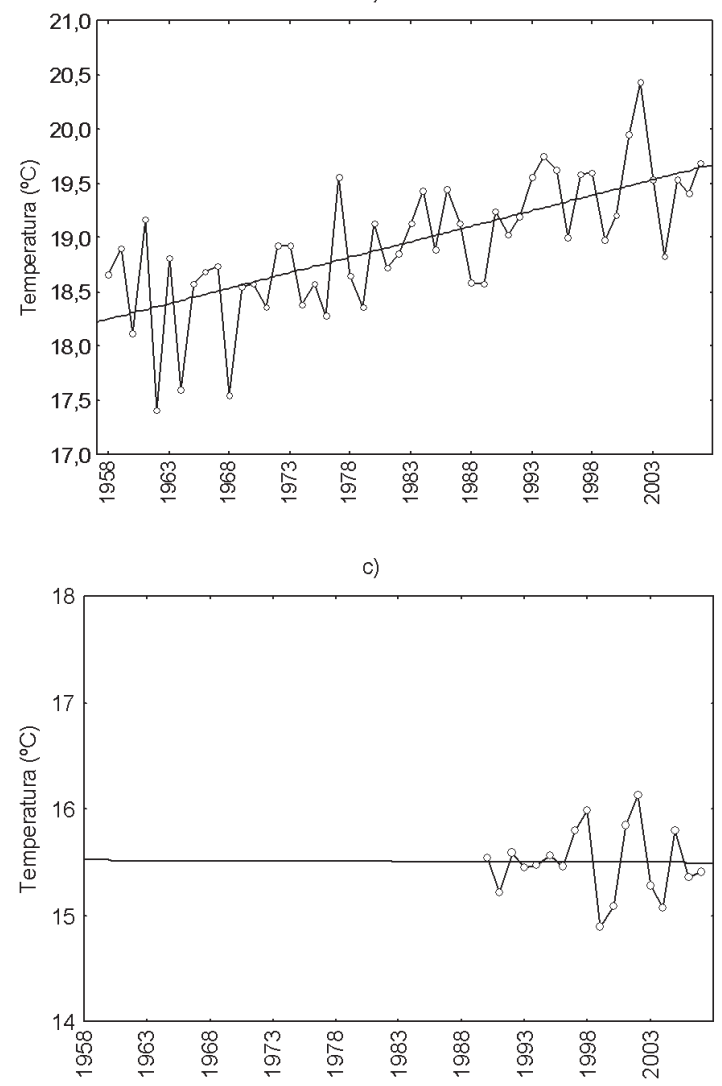

b)

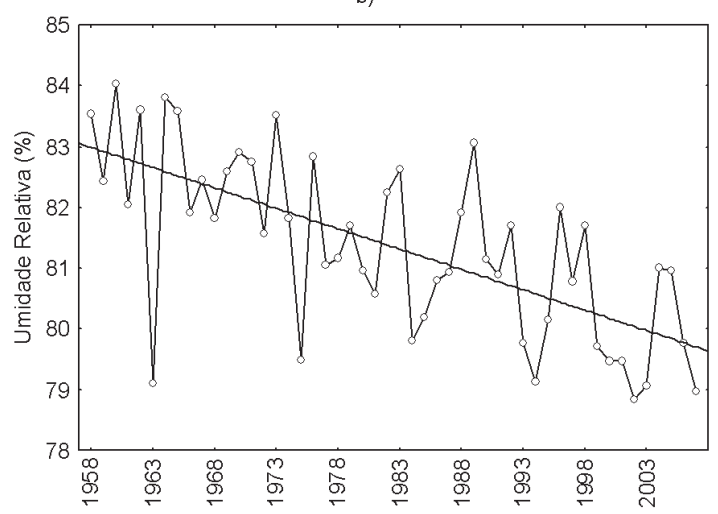

d)

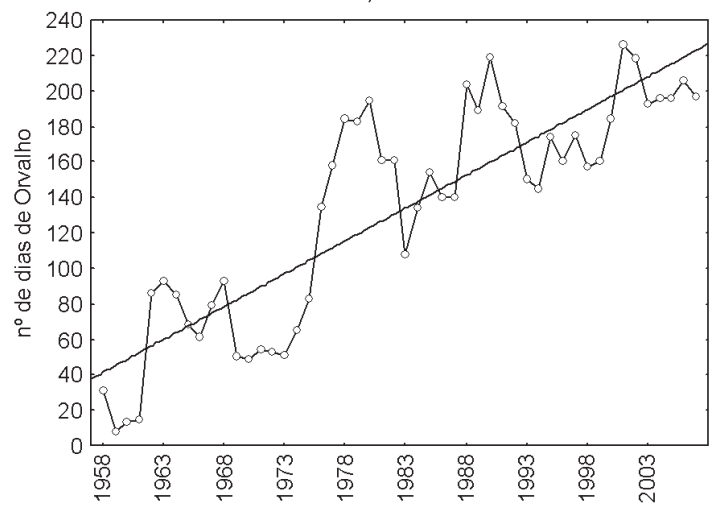

FIGURA 3 - a) Variação anual da temperatura média (série 1958-2007); b) Variação anual da umidade relativa média (série 1958-2007); c) Variação anual da temperatura do ponto de orvalho média (série 1990-2007); d) Variação do total anual de ocorrência de orvalho (série 1958-2007).

TABELA 1 - Equações de regressão e coeficientes de determinação e correlação de Pearson para D, T e UR, na série 1958-2007 (obs.: para $1958 \mathrm{x}=1$ e para 2007 $\mathrm{x}=50)$.

\begin{tabular}{ccc}
\hline $\begin{array}{c}\text { Equação de } \\
\text { regressão }\end{array}$ & $\begin{array}{c}\text { Coef. Det. } \\
\left(\mathrm{R}^{2}\right)\end{array}$ & $\begin{array}{c}\text { Coef. Corr. } \\
(\mathrm{R})\end{array}$ \\
\hline $\mathrm{D}=37,771+3,7039 \times$ & 0,7376 & 0,8588 \\
$\mathrm{~T}=18,219+0,0285 \times$ & 0,4776 & 0,6911 \\
$\mathrm{UR}=83,054-0,067 \times$ & 0,4501 & $-0,6709$ \\
\hline
\end{tabular}

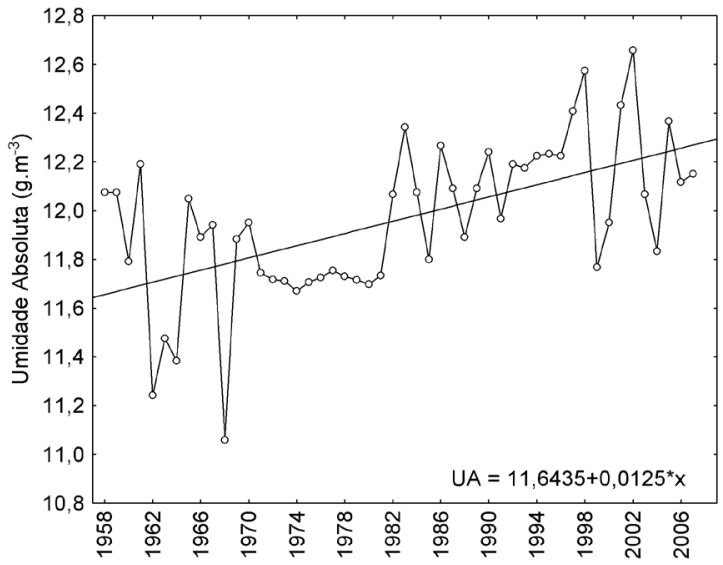

FIGURA 4 - Variação anual da Umidade Absoluta em São Paulo, no período 1958 a 2007. 

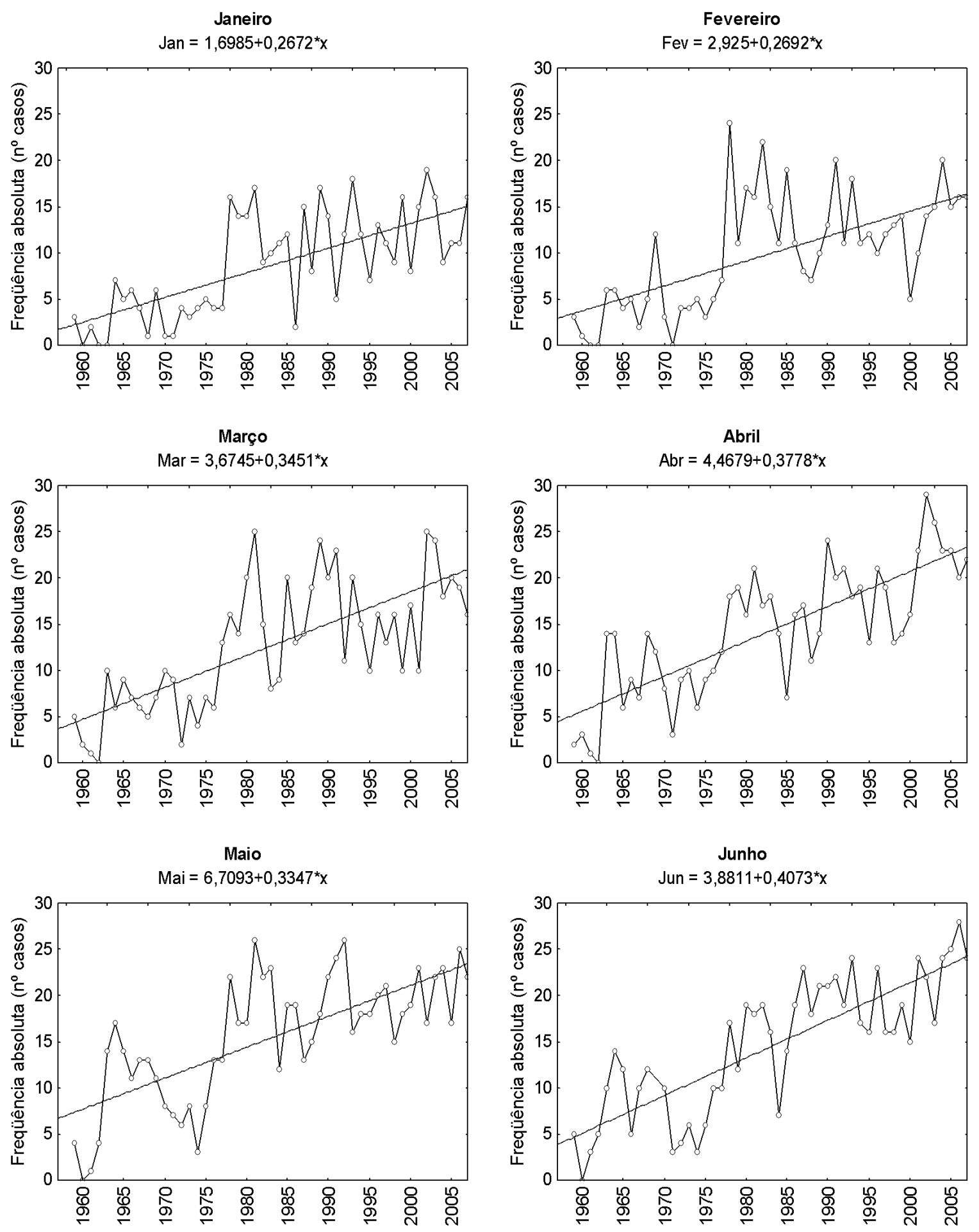

FIGURA 5A - Tendência do orvalho na série 1958-2007 (1 ${ }^{\circ}$ semestre). 

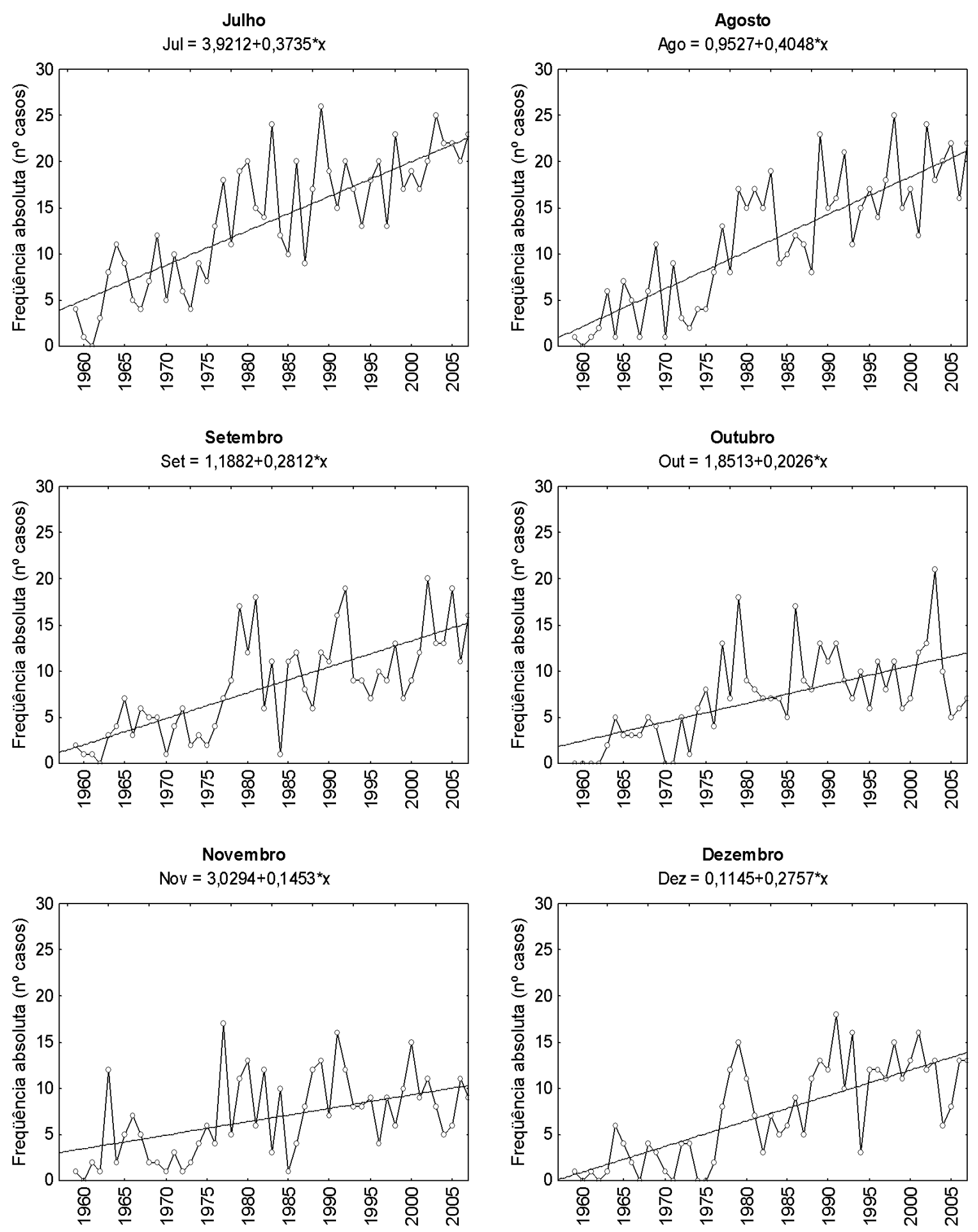

FIGURA 5B - Tendência do orvalho na série 1958-2007 (2ºmestre). 
A partir da análise da tendência anual de $\mathrm{T}$ (a), UR (b), Td (c), e D (d) (Figura 3), constata-se que $\mathrm{D}$ e $\mathrm{T}$ aumentaram ao longo dos anos e UR diminuiu. Na análise de variância, obteve-se elevada significância estatística e probabilidade de erro praticamente nula $(p=0,0000$ - ressalta-se que um algarismo diferente de zero apareceria somente após a $5^{\mathrm{a}}$ casa decimal) para a tendência de $\mathrm{T}$ e UR decorrente do tamanho da amostra $(\mathrm{N}=18.261$ dias transcorridos de 1 janeiro de 1958 a 31 de dezembro de 2007). FUNARI et al. (2008) apontaram para essa tendência, sem no entanto considerarem a amostra de dados diários e a probabilidade de erros envolvida.

As equações de regressão linear e os coeficientes de determinação para T, D e UR (Tabela 1) indicam que o número de dias com orvalho aumenta à razão de 3,7 dias por ano; a $\mathrm{T}$ aumenta $0,0285^{\circ} \mathrm{C}$ por ano e a UR decresce $0,067 \%$ por ano. Td manteve-se estável, muito embora se deva considerar que a série utilizada na análise é menor (1990-2007).

Analisando esse conjunto de variáveis verifica-se que as tendências da UR e de T são opostas ao longo dos anos, ou seja, enquanto $\mathrm{T}$ aumentou UR diminuiu. Este fato também foi constatado em análise estatística realizada por PEREIRA FILHO et al. (2007), que estudaram o período 1936-2005 da mesma Estação Meteorológica. Essa redução da umidade pode ter favorecido os resfriamentos noturnos, facilitando ou contribuindo para a ocorrência do orvalho, que apresentou tendência de aumento semelhante à de $\mathrm{T}$ e inversa à de UR. Também é possível notar que Td, com uma série mais curta (1990 a 2007), manteve-se estável, sugerindo que o aumento no orvalho está mais relacionado às variações de $T$ e UR.

Em função desses fatos, pode-se também levantar a hipótese de que esteja ocorrendo um aumento na quantidade de vapor de água na atmosfera, sobre a cidade de São Paulo, fato que contribuiria para justificar em parte o aumento observado no orvalho. A UA também apresentou tendência de aumento nestes 50 anos estudados (Figura 4), indicando ocorrência de aumento na quantidade de água na atmosfera que está sobre a cidade, que igualmente pode se condensar sob a forma de orvalho. Esse aumento de vapor de água na atmosfera urbana de São Paulo pode estar relacionado a um ciclo natural, mas provavelmente é atribuído à dinâmica e funcionamento da cidade, principalmente da queima de combus- tíveis fósseis, pois toda a combustão gera como produto água.

Em todos os meses do ano na série analisada está ocorrendo aumento do número de dias com formação de orvalho. No entanto, este fato é mais significativo no outono (abril, maio e junho), sendo seguido pelo inverno, fato que coincide com a maior frequência de orvalho nesses meses (Figura 1). Assim, o outono não apenas é a estação com maior frequência de orvalho, mas também a que apresenta a maior tendência de aumento nos últimos 50 anos, conforme pode ser observado nas figuras $5 \mathrm{a}$ e $5 \mathrm{~b}$.

\section{CONCLUSÕES}

A análise dos dados evidencia um aumento no número de dias com ocorrência de orvalho, sendo que os meses de abril a julho apresentam maior incidência e tendência de aumento desse fenômeno, resultado em consonância com aqueles de SERRA (1977) e FUNARI et al. (2008). Constatou-se que a maior frequência de dias de orvalho ocorre com UR média diária na faixa de $80 \%$ a $86 \%$, com UR máxima entre 95 e $98 \%$ e a mínima entre 37 e $62 \%$. Verificou-se, ainda, com o curso do tempo, diminuição da umidade relativa, fato este também já constatado por PEREIRA FILHO et al. (2007). Isso está associado ao aumento do número de dias de orvalho e da temperatura. Além disso, o aumento do orvalho também está relacionado ao aumento da umidade absoluta na atmosfera urbana da cidade de São Paulo nos últimos 50 anos, fato este relacionado ao processo de urbanização, mas, principalmente, ao "metabolismo" urbano.

A despeito dos resultados obtidos, salienta-se que mais apropriado seria trabalhar com os dados de temperatura de relva (inexistentes nessa estação meteorológica), pois é esse dado que ajudaria a compreender a formação de orvalho e as relações com os outros elementos climáticos utilizados neste trabalho.

\section{AGRADECIMENTOS}

Agradecemos à Estação Meteorológica do IAG-USP, pelo gentil fornecimento dos dados utilizados neste trabalho.

\section{REFERÊNCIAS BIBLIOGRÁFICAS}

AYOADE, J.O. 1983. Introdução à climatologia para os trópicos. Bertrand, Rio de Janeiro, 332 p. 
DUVDEVANI, S. 1947. An optical method of the dew estimation. Quartely Journal of Royal Meteorological Society, 73:282-296.

FRANÇA, A. 1946. Estudo sobre o clima na Bacia de São Paulo. Faculdade de Filosofia Ciências e Letras, Universidade de São Paulo, São Paulo, Tese de Doutorado, 59 p.

FRITSCHEN, L.J. 1963. Condensation of Shielded Net Radiometer. Journal of Applied Meteorology, 2:308-310.

FUNARI, F.L.; ARMANI, G; SALUM, S.T. 2008. Freqüência de orvalho na cidade de São Paulo. In: ABClima, SIMPÓSIO BRASILEIRO DE CLIMATOLOGIA GEOGRÁFICA, 8, Alto Caparaó, Anais, 429-436 (CD-ROM).

MONTEIRO, C.A.F. 1976. Teoria e clima urbano. São Paulo: Instituto de Geografia - USP, ), 179 p. (Série Teses e Monografias, 25).
MONTEIRO, C.A.F. 2003. Teoria e clima urbano: um projeto e seus caminhos. In: C.A.F. MONTEIRO \& F. MENDONÇA (ed.) Clima urbano. São Paulo, Contexto, p. 9-67.

MONTEITH, J.L. 1957. Dew. Quartely Journal of Royal Meteorological Society, 83: 322-341.

PEREIRA FILHO, A.J.; MARQUES DOS SANTOS, P.; XAVIER, T.M.B.S. 2007. Evolução do Tempo e Clima na Região Metropolitana de São Paulo. Linear B, IAG-USP, São Paulo, $282 \mathrm{p}$.

SERRA, A.B. 1969. Atlas Climatológico do Brasil (mapas selecionados). Escritório de Meteorologia, Rio de Janeiro.

SERRA. A.B. 1977. Orvalho - Geada. In: A.B. Serra, Climatologia do Brasil. Rio de Janeiro: CNPQ, 29p. (Fascículo 25).

Endereço dos autores:

Gustavo Armani - Seção de Hidroclimatologia, Instituto Geológico, Secretaria do Meio Ambiente do Estado de São Paulo, Avenida Miguel Stéfano, 3900, CEP 04301-903, Água Funda, São Paulo, SP. E-mail: gustavo@igeologico.sp.gov.br

Frederico Luiz Funari - Instituto de Astronomia, Geofísica e Ciências Atmosféricas da Universidade de São Paulo. Avenida Miguel Stéfano, 4200, CEP 04301-904, Água Funda, São Paulo, SP. E-mail: ffunari@ uol.com.br

Sergio Torre Salum - Estação Meteorológica, Instituto de Astronomia, Geofísica e Ciências Atmosféricas da Universidade de São Paulo. Avenida Miguel Stéfano, 4200, CEP 04301-904, Água Funda, São Paulo, SP. E-mail: estacao@model.iag.usp.br 